CALT $68-2877$

BRX-TH-658

\title{
Partial Masslessness and Conformal Gravity
}

\author{
S. Deser ${ }^{\sharp}$, E. Joung ${ }^{b}$ And A. WALDron ${ }^{\natural}$ \\ ${ }^{\sharp}$ Lauritsen Lab, Caltech, Pasadena CA 91125 and Physics Department, Brandeis \\ University, Waltham, MA 02454, USA \\ deser@brandeis.edu \\ b Scuola Normale Superiore and INFN, Piazza dei Cavalieri 7, 56126 Pisa, Italy \\ euihun.joung@sns.it \\ ${ }^{\natural}$ Department of Mathematics, University of California, Davis, CA 95616, USA \\ wally@math.ucdavis.edu
}

\begin{abstract}
We use conformal, but ghostful, Weyl gravity to study its ghost-free, second derivative, partially massless (PM) spin 2 component in presence of Einstein gravity with positive cosmological constant. Specifically, we consider both gravitational- and self- interactions of PM via the fully non-linear factorization of conformal gravity's Bach tensor into Einstein times Schouten operators. We find that extending PM beyond linear order suffers from familiar higher-spin consistency obstructions: it propagates only in Einstein backgrounds, and the conformal gravity route generates only the usual safe, Noether, cubic order vertices.
\end{abstract}

PACS: $04.62 .+\mathrm{v}, 04.50 .-\mathrm{h}, 04.50 . \mathrm{Kd}, 04.62 .+\mathrm{v}, 02.40 .-\mathrm{k}$ 


\section{Introduction and Review}

Conformal, $d=4$, Weyl gravity $(\mathrm{CG})$ provides a natural arena for studying the partially massless 1 (PM) spin-2 field: when expanded about de Sitter (dS) backgrounds, its kinematics consist of PM and the graviton [7]. Indeed, PM is invariant under the (tuned) sum of (linearized) diffeomorphism and conformal transformations:

$$
\delta \varphi_{\mu \nu}=\left(\nabla_{\mu} \partial_{\nu}+\frac{\Lambda}{3} g_{\mu \nu}\right) \alpha(x) .
$$

In particular, we will use CG to test whether the well-known gravitationaland self- coupling difficulties encountered by higher-spin fields can be circumvented for PM, while avoiding the physically unacceptable ghost excitations CG shares with all higher derivative models. We will find first that while PM can live in Einstein (Einstein tensor proportional to the metric), rather than just dS (constant curvature) spaces [1, 2, 8], it breaks down in more general classes of geometries. We then find that although PM's self-coupling still faces the problems of higher-spin fields, in dS or Einstein backgrounds self-interacting cubic vertices can be obtained by contracting a Noether current with its corresponding gauge field. Consistency beyond cubic order is, however, difficult to maintain. CG avoids this pitfall at the cost of ghost excitations, but its truncation to ghost-free PM modes fails beyond its lowest, cubic, order.

Interactions of PM in four dimensions in particularly interesting because it is rigidly $S O(4,2)$ conformally invariant [9], just like its vector Maxwell counterpart. In fact PM can be coupled to charged matter fields [10] (see also [11]) which suggests forming non-abelian multiplets-a still open problem. Instead, we will be concerned with the natural (singlet) tensor analogy and (abelian) self-interactions possibility. Results for cubic vertices derived from a Stückelberg approach were first given in [12] while a more general calculus of higher derivative PM cubic vertices was developed in [13]. Also, it has recently been suggested that a PM limit of the putative massive gravity could be a candidate for an interacting PM theory [14].

\footnotetext{
${ }^{1}$ Recall that there are three varieties of spin 2 excitations in dS: massive, massless and partially massless [1, 2] The latter enjoy an interesting mixed behavior: In dS they propagate lightlike, positive energy (inside the maximally accessible intrinsic dS horizon), helicity $\pm 2, \pm 1$ excitations in a unitary representation of the dS isometry group [3, 5, 4, 6]. This degree of freedom (DoF) count relies on the gauge invariance (11) and a divergence constraint $\nabla^{\mu} \varphi_{\mu \nu}=\nabla_{\nu} \varphi$, where $\varphi:=\varphi_{\rho}^{\rho}$.
} 
Weyl transformations underlie PM's invariances, so CG is the obvious tool for studying its interactions. While CG always has six excitations, the detailed spectra are background-dependent. About flat space, it has two massless tensors and a photon with the same signature as one of them [15], while in constant curvature backgrounds there is still a (cosmological) graviton, but now the (tensor+photon) combination becomes the PM mode with helicities $( \pm 2, \pm 1)$. In each case, the two sets of modes are relatively ghostlike. The relative sign between PM's helicities depends on that of $\Lambda$ : In anti de Sitter (AdS), one can truncate the solution space to just the unitary, massless graviton [7, 16, 17] (for related analysis of higher derivative theories see [18, 19]). The dS story is the interesting one here because we can truncate, leaving either mode unitary; the truncation to a unitary, PM mode being the relevant one.

A central ingredient of CG is the Yang-Mills theory of the conformal group $S O(4,2)$; it underlies an Ostrogradski treatment in terms of a second derivative order action [20] with field content directly corresponding to gravitons and PM. It is also the starting point for modern mathematical approaches to conformal geometries [21]. Indeed, given the following gravity data: (i) the vierbein $e_{\mu}^{m}$, (ii) the Levi-Civita connection $\nabla_{\mu}$ and (iii) the Schouten tensor $\mathrm{P}_{\mu \nu}:=\frac{1}{2}\left(R_{\mu \nu}-\frac{1}{6} g_{\mu \nu} R\right)$, then the particular $S O(4,2)$ YangMills connection

$$
\nabla_{\mu}^{\mathcal{T}}=\mathbb{I} \nabla_{\mu}+\left(\begin{array}{ccc}
0 & -e_{\mu}{ }^{n} & 0 \\
\mathrm{P}_{\mu}^{m} & 0 & e_{\mu}{ }^{m} \\
0 & -\mathrm{P}_{\mu}^{n} & 0
\end{array}\right)
$$

plays a distinguished rôle. It is called the tractor connection [21]. Any connection $\nabla$ faces three fundamental questions:

1. When does its curvature $F=\nabla^{2}$ vanish?

2. When are there parallel sections: vectors $I$ such that $\nabla I=0$ ?

3. When does $F$ obey the Yang-Mills equations $\nabla^{*} F=0$ ?

The answers for the tractor connection $\nabla^{\mathcal{T}}$ on a conformal 4-manifold $\left(M,\left[g_{\mu \nu}\right]\right)$ (one equipped with a conformal class of metrics $\left[g_{\mu \nu}\right]=\left[\Omega^{2} g_{\mu \nu}\right]$ ) are:

1. When $g_{\mu \nu}$ is conformally flat [21].

2. When $g_{\mu \nu}$ is conformal to an Einstein metric [21]. 
3. When $g_{\mu \nu}$ is Bach-flat 22] (i.e. a CG solution, see (33)).

While Einstein metrics are of prime importance, our main focus will be on Bach-flat metrics, since vanishing of the Bach tensor is the CG equation of motion that follows from the action

$$
S[g]=\frac{1}{8} \int \sqrt{-g} W^{\mu \nu \rho \sigma} W_{\mu \nu \rho \sigma}=\frac{1}{4} \int \sqrt{-g}\left(R^{\mu \nu} R_{\mu \nu}-\frac{1}{3} R^{2}\right),
$$

modulo the Euler invariant.

The above three conditions ascend in generality: conformally flat metrics are conformally Einstein and conformally Einstein metrics are Bach-flat. A key question is how to characterize Bach-flat metrics that are not conformally Einstein. An answer involves PM fields in an essential way: Consider the, symmetric, trace- and divergence-free Bach tensor

$$
B_{\mu \nu}:=-\Delta \mathrm{P}_{\mu \nu}+\nabla^{\rho} \nabla_{(\mu} \mathrm{P}_{\nu) \rho}+W_{\rho \mu \nu \sigma} \mathrm{P}^{\rho \sigma}
$$

which is invariant under local Weyl rescaling:2

$$
\delta g_{\mu \nu}=2 \alpha g_{\mu \nu} \quad \Rightarrow \quad \delta \mathrm{P}_{\mu \nu}=-\nabla_{\mu} \partial_{\nu} \alpha
$$

Using the Bianchi identity

$$
\nabla^{\nu} \mathrm{P}_{\mu \nu}=\nabla_{\mu} \mathrm{P}, \quad \mathrm{P}:=\mathrm{P}_{\rho}^{\rho}
$$

reordering covariant derivatives at the cost of terms quadratic in $\mathrm{P}_{\mu \nu}$, and defining the cosmological Schouten tensor

$$
\varphi_{\mu \nu}:=-\mathrm{P}_{\mu \nu}+\frac{\Lambda}{6} g_{\mu \nu}
$$

the Bach tensor reads

$$
\begin{aligned}
B_{\mu \nu}(g, \varphi)= & \Delta \varphi_{\mu \nu}-2 \nabla_{(\mu} \nabla^{\rho} \varphi_{\nu) \rho}+g_{\mu \nu} \nabla^{\rho} \nabla^{\sigma} \varphi_{\rho \sigma}+\nabla_{\mu} \nabla_{\nu} \varphi-g_{\mu \nu} \Delta \varphi \\
& -2 W_{\rho \mu \nu \sigma} \varphi^{\rho \sigma}-\frac{4}{3} \Lambda\left(\varphi_{\mu \nu}-\frac{1}{4} g_{\mu \nu} \varphi\right)+O\left(\varphi^{2}\right) .
\end{aligned}
$$

If $g_{\mu \nu}$ is close to an Einstein metric with cosmological constant $\Lambda$, it follows that $\mathrm{P}_{\mu \nu} \approx \frac{\Lambda}{6} g_{\mu \nu}$, so $\varphi_{\mu \nu}$ can be viewed a fluctuation and its quadratic terms

\footnotetext{
${ }^{2}$ In dimension three, the 2-index form of the Weyl-invariant, traceless and conserved, Cotton tensor, $C_{\mu \nu}{ }^{m}:=\nabla_{\mu} \mathrm{P}_{\nu}{ }^{m}-\nabla_{\nu} \mathrm{P}_{\mu}{ }^{m}$ generates the "Weyl"-CS model [23].
} 
can, to leading order, be dropped. Upon setting $g_{\mu \nu}$ to an Einstein metric, the resulting linear equation for $\varphi_{\mu \nu}$ is exactly the PM field equation in the Einstein background. In particular, observe that the Weyl transformation formula (41) for the Schouten tensor yields the gauge invariance (11) of the PM field $\varphi_{\mu \nu}$ : the cosmological Schouten tensor corresponds to the PM field in CG.

The fact that CG yields the PM equations in Einstein spaces motivates our two main questions:

1. Can PM fields be coupled to gravitational backgrounds more general than Einstein?

2. Can CG be used as a catalyst to generate consistent, ghost-free, selfinteractions for PM fields, at least in Einstein backgrounds?

Our answer to the first question is given in Section 2. There we review results from conformal geometry that completely characterize the failure of generic Bach-flat metrics (CG solutions) to be Einstein (cosmological Einstein gravity solutions). This gives the technology to study propagation of free PM fields in general backgrounds. We consider, then disprove the natural conjecture that Bach-flat backgrounds support consistent propagation of PM fields.

In Section 3 we review the truncation of CG to cosmological Einstein gravity by turning off its PM excitations. Then we consider the converse question: to what extent can the the configuration space of $\mathrm{CG}$ be split into PM and graviton fields such that the dynamical metric can be held to a fixed Einstein background, leaving a theory of interacting PM fields? While this mechanism holds at linear level for the field equations, we show it cannot be continued to non-linear interactions. This does not mean that no self-interacting PM model exists, but rather that it could not be a CGtruncation. CG can still be used to generate cubic vertices that are consistent with the leading order PM gauge invariance. While the construction of cubic order vertices says nothing about the existence of a fully interacting theory, this mechanism generates them efficiently.

In the Conclusion, we summarize our results and discuss to what extent our CG based no-go statements extend to general PM theories. We also speculate briefly about novel approximate cosmological solutions to Einstein gravity generated by a CG-dominated epoch, and also on higher-spin adaptations of our approach. 


\section{PM-Gravitational Interactions}

A natural (but soon to be disproved!) conjecture is that Bach-flat backgrounds are the most general ones in which (linear) PM fields propagate. Striking evidence for this is the existence of Weyl invariant field equations enjoying double derivative gauge invariances precisely when the background is Bach-flat. These models derive from ideas in conformal geometry: Let us return to the parallel condition $\nabla^{\mathcal{T}} I=0$ for the tractor connection defined in Section 1. Denoting the so-called scale tractor by $I_{M}=\left(\rho, n_{\mu}, \sigma\right)$, this condition becomes [21]

$$
\left\{\begin{array}{c}
\partial_{\mu} \sigma-n_{\mu}=0 \\
\nabla_{\mu} n_{\nu}+\sigma \mathrm{P}_{\mu \nu}+\rho g_{\mu \nu}=0 \\
\partial_{\mu} \rho-\mathrm{P}_{\mu \nu} n^{\nu}=0
\end{array}\right.
$$

This system enjoys (local) conformal invariance; in particular, under local Weyl rescalings, $\sigma \mapsto e^{\alpha} \sigma$, so setting $\sigma=1$ is a legal gauge choice. Then the first equation gives $n_{\mu}=0$, the second equation states $\mathrm{P}_{\mu \nu} \propto g_{\mu \nu}$ : the parallel condition implies that the metric is (conformally) Einstein. (The converse is also true, see [21]). Returning to a general gauge and using the first and third equations we can express the scale tractor $I_{M}$ in terms of $\sigma$, as $I_{M}=\left(-\frac{1}{4}(\Delta+\mathrm{P}) \sigma, \nabla_{\mu} \sigma, \sigma\right)$. The parallel conditions now reduce to

$$
\boldsymbol{P}_{\mu \nu} \sigma=0
$$

where the operator $\boldsymbol{P}_{\mu \nu}$, which maps scalars to trace-free symmetric tensors, is in fact conformally invariant. Explicitly

$$
\boldsymbol{P}_{\mu \nu}:=\nabla_{\{\mu} \partial_{\nu\}}+\mathrm{P}_{\{\mu \nu\}},
$$

where $X_{\{\mu \nu\}}:=X_{(\mu \nu)}-\frac{1}{4} g_{\mu \nu} X_{\rho}^{\rho}$ is the symmetrized trace-free part of $X_{\mu \nu}$, so $g^{\mu \nu} X_{\{\mu \nu\}}=0$. Zero modes of this operator correspond to conformally Einstein metrics; if $\sigma \in \operatorname{ker} \boldsymbol{P}_{\mu \nu}$, then $\sigma^{-2} g_{\mu \nu}$ is an Einstein metric [24]. In addition, the gauge transformation of the trace-free components of a PM field $\varphi_{\mu \nu}$ are generated by $\boldsymbol{P}_{\mu \nu}$. The key property, for our purposes, of the operator $\boldsymbol{P}_{\mu \nu}$ was discovered in [24]: it permits the operator factorization of the Bach tensor as

$$
B_{\mu \nu}=\boldsymbol{M}_{\mu \nu}^{\rho \sigma} \boldsymbol{P}_{\rho \sigma}, \quad \boldsymbol{M}_{\mu \nu}^{\rho \sigma}:=\delta_{\{\mu}^{\rho} \delta_{\nu\}}^{\sigma} \Delta-\delta_{\{\mu}^{\rho} \nabla^{\sigma} \nabla_{\nu\}}-\frac{1}{3} \delta_{\{\mu}^{\rho} \nabla_{\nu\}} \nabla^{\sigma}-W_{\mu \nu}^{\rho}{ }_{\mu}^{\sigma}
$$


We observe that $\boldsymbol{M}_{\mu \nu}^{\rho \sigma}$ gives the non-linear answer to the question posed in the Introduction: characterizing Bach-flat metrics that are not conformally Einstein. We see that those require the range of $\boldsymbol{P}_{\mu \nu}$ to intersect the kernel of $\boldsymbol{M}_{\mu \nu}$; the operator $\boldsymbol{M}$ is also conformally invariant and maps trace-free symmetric tensors to trace-free symmetric tensors.

In mathematical terms, the identity (77) means that the sequence of three differential operators $\boldsymbol{P}_{\rho \sigma}, \boldsymbol{M}_{\mu \nu}^{\rho \sigma}, \boldsymbol{P}^{\dagger \mu \nu}$ (the third being the adjoint of $\boldsymbol{P}_{\mu \nu}$ ) form a complex whenever the metric is Bach-flat. In [24], this is termed a Yang-Mills detour complex. Physically, it implies that the field equation

$$
M_{\mu \nu}^{\rho \sigma} \widetilde{\varphi}_{\rho \sigma}=\Delta \widetilde{\varphi}_{\mu \nu}-\nabla^{\sigma} \nabla_{\{\mu} \widetilde{\varphi}_{\nu\} \sigma}-\frac{1}{3} \nabla_{\{\mu} \nabla^{\sigma} \widetilde{\varphi}_{\nu\} \sigma}-W_{\mu \nu}^{\rho}{ }^{\sigma} \widetilde{\varphi}_{\rho \sigma}=0,
$$

for a trace-free symmetric tensor $\widetilde{\varphi}_{\mu \nu}=: \varphi_{\{\mu \nu\}}$, enjoys the double derivative gauge invariance (and associated double derivative Bianchi identity)

$$
\delta \widetilde{\varphi}_{\mu \nu}=\boldsymbol{P}_{\mu \nu} \alpha=\left(\nabla_{\{\mu} \nabla_{\nu\}}+\widetilde{\mathrm{P}}_{\mu \nu}\right) \alpha
$$

in Bach-flat backgrounds. This was the motivation for our original conjecture that PM fields could propagate in them. We now proceed to disprove it and give necessary consistency conditions for PM-compatible backgrounds.

The Bach tensor, since it arises from a metric variational principle, is necessarily divergence-free, $\nabla^{\mu} \boldsymbol{M}_{\mu \nu}^{\rho \sigma} \boldsymbol{P}_{\rho \sigma}=0$. However, it is neither true that $\nabla^{\mu} \boldsymbol{M}_{\mu \nu}^{\rho \sigma}=0$, nor that $\nabla^{\mu} \boldsymbol{M}_{\mu \nu}^{\rho \sigma}=O(\nabla)$ (rather this operator is cubic in derivatives). But consistent PM propagation relies on a divergence constraint3; for a PM field equation (derived from an action) this requirement is precisely expressed by the condition $\nabla^{\mu} \boldsymbol{M}_{\mu \nu}^{\rho \sigma}=O(\nabla)$.

The failure of the field equation (8) to imply an appropriate divergence constraint does not yet rule out PM fields interacting with backgrounds more general than Einstein spaces, because we may still enlarge the space of field equation and gauge operators, $\boldsymbol{M}_{\mu \nu}^{\rho \sigma}$ and $\boldsymbol{P}_{\mu \nu}$ respectively, by relaxing their trace-free and conformal invariance properties. To test that we make the following generalization

$$
\begin{aligned}
\boldsymbol{M}_{\mu \nu}^{\prime \rho \sigma} & =\boldsymbol{G}_{\mu \nu}^{\rho \sigma}-\left(\delta_{(\mu}^{\rho} \delta_{\nu)}^{\sigma}-g_{\mu \nu} g^{\rho \sigma}\right) \mathrm{P}+\alpha_{1} \delta_{(\mu}^{\rho} \widetilde{\mathrm{P}}_{\nu)}^{\sigma}+\alpha_{2}\left(g_{\mu \nu} \widetilde{\mathrm{P}}^{\rho \sigma}+\widetilde{\mathrm{P}}_{\mu \nu} g^{\rho \sigma}\right), \\
\boldsymbol{P}_{\mu \nu}^{\prime} & =\nabla_{\mu} \partial_{\nu}+\frac{1}{2} \mathrm{P} g_{\mu \nu}+\beta \widetilde{\mathrm{P}}_{\mu \nu},
\end{aligned}
$$

\footnotetext{
${ }^{3}$ The DoF count for PM starts with ten off-shell fields $\varphi_{\mu \nu}$, minus four DoF thanks to the divergence constraint $\nabla^{\mu} \varphi_{\mu \nu}=\nabla_{\nu} \varphi$, minus two further DoF by the scalar gauge invariance, yielding a total of four on-shell excitations.
} 
where the cosmological Einstein operator

$$
\begin{aligned}
\boldsymbol{G}_{\mu \nu}^{\rho \sigma}:= & \left(\delta_{(\mu}^{\rho} \delta_{\nu)}^{\sigma}-g_{\mu \nu} g^{\rho \sigma}\right)(\Delta-\mathrm{P})-2 \nabla_{(\mu} \nabla^{\rho} \delta_{\nu)}^{\sigma}+\nabla_{(\mu} \nabla_{\nu)} g^{\rho \sigma}+g_{\mu \nu} \nabla^{\rho} \nabla^{\sigma} \\
& -2 W^{\rho}{ }_{\mu \nu}{ }^{\sigma}-8 \widetilde{\mathrm{P}}_{\{\mu}^{\rho} \delta_{\nu\}}^{\sigma}-\frac{3}{2} g_{\mu \nu} \mathrm{P} g^{\rho \sigma}
\end{aligned}
$$

is identically conserved

$$
\nabla^{\mu} \boldsymbol{G}_{\mu \nu}^{\rho \sigma}=0
$$

in Einstein backgrounds. The equation of motion of cosmological Einstein gravity linearized about an Einstein metric is $\boldsymbol{G}_{\mu \nu}^{\rho \sigma} \varphi_{\rho \sigma}=0$.

The above ansatz is the most general one obeying the following requirements:

1. The operators $\boldsymbol{M}_{\mu \nu}^{\prime \rho \sigma}$ and $\boldsymbol{P}_{\mu \nu}^{\prime}$ are second order in $\nabla$ or derivatives on the metric $g_{\mu \nu}$.

2. The operator $\boldsymbol{M}_{\mu \nu}^{\rho \sigma}$ is self-adjoint, to ensure the existence of an action principle.

3. The divergence $\nabla^{\mu} \boldsymbol{M}_{\mu \nu}^{\prime \rho \sigma}$ is an operator no more than linear in $\nabla$, to ensure that solutions of $\boldsymbol{M}_{\mu \nu}^{\prime \rho \sigma} \varphi_{\mu \nu}=0$ obey a first order constraint.

4. The operator product $\boldsymbol{M}_{\mu \nu}^{\prime \rho \sigma} \boldsymbol{P}_{\rho \sigma}^{\prime}$ vanishes when $g_{\mu \nu}$ is an Einstein metric; this fixes their leading terms to be operators corresponding to the linear PM equation of motion (6) and its double derivative gauge invariance (11). The remaining freedom in the ansatz therefore depends only on the trace-free Schouten tensor $\widetilde{\mathrm{P}}_{\mu \nu}$, since that quantity vanishes for Einstein metrics.

It remains to compute the product $\boldsymbol{M}_{\mu \nu}^{\prime \rho \sigma} \boldsymbol{P}_{\rho \sigma}^{\prime}$. The result can be arranged as an expansion in the gradient operator $\nabla$. By construction, terms of order $\nabla^{4}$ and $\nabla^{3}$ necessarily vanish. Prefactors of the terms order $\nabla^{2}$ only involve $\widetilde{\mathrm{P}}_{\mu \nu}$ which we are now assuming to be non-vanishing, since we wish to investigate metrics that are not Einstein: we must choose the constants $\left(\alpha_{1}, \alpha_{2}, \beta\right)$ accordingly and find

$$
\alpha_{1}=4+2 \beta \quad \text { and } \quad \alpha_{2}=-\beta .
$$

The analysis of terms order $\nabla$ and lower is more complicated. First we consider the trace $g^{\mu \nu} \boldsymbol{M}_{\mu \nu}^{\prime \rho \sigma} \boldsymbol{P}_{\rho \sigma}^{\prime}$ at order $\nabla$ and find $3 \beta\left(\nabla_{\rho} \mathrm{P}\right) \nabla^{\rho}$. There are 
two possibilities, either $\beta=0$ or the background metric has constant scalar curvature. Since the latter would rule out the PM conjecture in question, we choose $\beta=0$. We then find $g^{\mu \nu} \boldsymbol{M}_{\mu \nu}^{\prime \rho \sigma} \boldsymbol{P}_{\rho \sigma}^{\prime}=-3(\Delta \mathrm{P})$, which requires the scalar curvature to be harmonic, and hence also rules out the conjecture.

Having disproved the conjecture, one may still investigate whether some background condition weaker than Bach-flat, but still less stringent than Einstein, could yield consistent propagation. The terms remaining at order $\nabla$ in $\boldsymbol{M}_{\mu \nu}^{\prime \rho \sigma} \boldsymbol{P}_{\rho \sigma}^{\prime}$ are

$$
\beta g_{\mu \nu}\left(\nabla_{\rho} \mathrm{P}\right) \nabla^{\rho}-(\beta-2)\left(\nabla_{(\mu} \mathrm{P}\right) \nabla_{\nu)}+2(\beta-1)\left(\nabla_{\rho} \mathrm{P}_{\mu \nu}\right) \nabla^{\rho}-2 \beta\left(\nabla_{(\mu} \mathrm{P}_{\nu) \rho}\right) \nabla^{\rho} ;
$$

clearly no choice of $\beta$ removes all of them. Instead, we can restrict the background, one option being to Ricci-symmetric spaces, defined by $\nabla_{\rho} \mathrm{P}_{\mu \nu}$ $=0$. This condition is weaker than Einstein, but need not imply Bach-flat. However, even then we must cancel all terms in $\boldsymbol{M}_{\mu \nu}^{\prime \rho \sigma} \boldsymbol{P}_{\rho \sigma}^{\prime}$ of order $\nabla^{0}$. In general backgrounds these are

$$
\begin{gathered}
-\beta B_{\mu \nu}+2 \beta^{2} \mathrm{P}_{(\mu}^{\rho} \mathrm{P}_{\nu) \rho}-\frac{1}{2}(\beta-1)(\beta+3) \mathrm{P}_{\mu \nu}-\frac{1}{2}(\beta-2) \nabla_{\mu} \partial_{\nu} \mathrm{P} \\
+g_{\mu \nu}\left[\frac{1}{2}(\beta-2) \Delta \mathrm{P}-\beta(\beta+1) \mathrm{P}_{\rho \sigma} \mathrm{P}^{\rho \sigma}+\frac{1}{8}(\beta+2)(3 \beta-2) \mathrm{P}^{2}\right] .
\end{gathered}
$$

Even for a Ricci-symmetric space, no choice of $\beta$ removes all remaining terms quadratic in the Schouten tensor and its trace. We also see no strong physical motivation to single out backgrounds with covariantly constant Einstein tensor subject to a further quadratic curvature constraint.

\section{Self-Interactions}

To study self-interactions, we must first recast our derivation of the PM field equation (66) in terms of the CG action (2). The latter can be rewritten as a two-derivative action by introducing an auxiliary field $\varphi_{\mu \nu}$ [20]:

$$
S[g, \varphi]=-\int \sqrt{-g}\left[\frac{\Lambda}{6}(R-2 \Lambda)+\varphi^{\mu \nu} \mathcal{G}_{\mu \nu}+\varphi^{\mu \nu} \varphi_{\mu \nu}-\varphi^{2}\right]
$$

where $\mathcal{G}_{\mu \nu}:=G_{\mu \nu}+\Lambda g_{\mu \nu}$ is the cosmological Einstein tensor. Upon completing the square, we see that the auxiliary field becomes the cosmological Schouten tensor (5). To analyze the spectrum of the theory about an Einstein background $\bar{g}_{\mu \nu}$ with cosmological constant $\Lambda$, we linearize in metric 
perturbations $h_{\mu \nu}=g_{\mu \nu}-\bar{g}_{\mu \nu}$. Keeping terms quadratic in fluctuations and making the field redefinition

$$
h_{\mu \nu} \rightarrow h_{\mu \nu}+\frac{\Lambda}{6} \varphi_{\mu \nu} .
$$

yields the action (the metrics appearing in $\boldsymbol{G}$ and $\boldsymbol{F}$ are set to $\bar{g}_{\mu \nu}$ )

$$
S^{(2)}[h, \varphi]=\frac{1}{4} \int \sqrt{-\bar{g}}\left[-\frac{\Lambda}{6} h^{\mu \nu} \boldsymbol{G}_{\mu \nu}^{\rho \sigma} h_{\rho \sigma}+\frac{6}{\Lambda} \varphi^{\mu \nu}\left(\boldsymbol{G}_{\mu \nu}^{\rho \sigma}-\frac{2}{3} \Lambda \boldsymbol{F}_{\mu \nu}^{\rho \sigma}\right) \varphi_{\rho \sigma}\right] .
$$

Here $-\boldsymbol{G}_{\mu \nu}^{\rho \sigma} h_{\rho \sigma} / 2$ is the linearized cosmological Einstein tensor defined in (9) and all indices are moved by $\bar{g}_{\mu \nu}$. The Pauli-Fierz $(\mathrm{PF})$ mass operator is defined as $\boldsymbol{F}_{\mu \nu}^{\rho \sigma}:=\delta_{\mu}^{\rho} \delta_{\nu}^{\sigma}-g_{\mu \nu} g^{\rho \sigma}$, so the PM field equation is

$$
\left(\boldsymbol{G}_{\mu \nu}^{\rho \sigma}-\frac{2}{3} \Lambda \boldsymbol{F}_{\mu \nu}^{\rho \sigma}\right) \varphi_{\rho \sigma}=0 .
$$

Thus, the first term of (12) is linearized Einstein-Hilbert, while the terms with round brackets (the sum of the linearized gravity kinetic term and a Pauli-Fierz mass term tuned to the PM value $m^{2}=2 \Lambda / 3$ ) give the PM theory, all in an Einstein background. Hence the model describes the "difference" of massless and PM excitations. Moreover, integrating out (at linear level) the field $\varphi_{\mu \nu}$ appearing before the field redefinition (11), gives the fourth order equation

$$
\boldsymbol{B}_{\mu \nu}^{\rho \sigma} h_{\rho \sigma}=0, \quad \text { where } \quad \boldsymbol{B}_{\mu \nu}^{\rho \sigma}:=\boldsymbol{G}_{\mu \nu}^{\alpha \beta} \boldsymbol{F}_{\alpha \beta}^{-1 \gamma \delta} \boldsymbol{G}_{\gamma \delta}^{\rho \sigma}-\frac{2}{3} \Lambda \boldsymbol{G}_{\mu \nu}^{\rho \sigma},
$$

for the original metric fluctuations. Indeed, $\boldsymbol{B}_{\mu \nu}^{\rho \sigma} h_{\rho \sigma}$ is the Bach tensor linearized about an Einstein background.

The relative sign of the two parts of the linearized action (12) reflects the unavoidable relative ghost structure. In particular, states with $\varphi_{\mu \nu}=0$ constitute a unitary, massless spin $s=2$ spectrum. When the cosmological constant is positive $(\mathrm{dS})$, states with $h_{\mu \nu}=0$ correspond to a unitary PM spectrum. We now proceed to study the latter truncation; a key step is to understand the model's gauge structure. At linear level, the graviton $h_{\mu \nu}$ enjoys a linearized diffeomorphism symmetry $\delta h_{\mu \nu}=\nabla_{\mu} \xi_{\nu}+\nabla_{\nu} \xi_{\mu}$ while the PM

\footnotetext{
${ }^{4}$ As an aside, we observe that the derivation of the linear PM model from Weyl invariant CG theory gives a novel proof of the $S O(4,2)$ conformal invariance of PM excitations. (In fact, conformal invariance was the original rationale behind the PM model [1, and is enjoyed by all maximal depth, four-dimensional PM theories of generic spin [9].) In detail, whenever a field is coupled to the metric, maintaining Weyl invariance, then setting the metric to a background yields an action that enjoys any conformal isometries as symmetries. Thus the non-linear model generated by setting the metric in (10) to a background is guaranteed to enjoy this symmetry; since it holds order by order in $\varphi$, it is also a symmetry of linearized PM.
} 
field $\varphi_{\mu \nu}$ transforms according to the double derivative scalar variation (1); at linear level each field is inert under the other's transformations. In fact, the PM gauge symmetry is inherited from the Weyl symmetry of CG. The full non-linear action (10) is invariant under both gauge transformations,

$$
\delta g_{\mu \nu}=\nabla_{\mu} \xi_{\nu}+\nabla_{\nu} \xi_{\mu}+2 \alpha g_{\mu \nu}, \quad \delta \varphi_{\mu \nu}=\mathcal{L}_{\xi} \varphi_{\mu \nu}+\left(\nabla_{\mu} \partial_{\nu}+\frac{\Lambda}{3} g_{\mu \nu}\right) \alpha
$$

The metric transformation is now a sum of diffeomorphism and Weyl transformations as is the $\varphi_{\mu \nu}$ transformation: $\mathcal{L}_{\xi}$ is the Lie derivative along the vector field $\xi$ and the Weyl term follows from the transformation of the Schouten tensor (4).

Without incurring the ghost problem of CG, we may search for some combination of fields that, when held to an appropriate background, yields a consistent truncation to a self-interacting PM model 5 We must now find the proper combination of fields to set to a background that yields the desired decoupling. At linear level, the answer to this requirement is given by the field redefinition (11). There, the choice for the metric fluctuations $h_{\mu \nu}=0$ is respected by PM gauge transformations. This substitution in the linearized action (12) yields the free PM action in an Einstein background. Therefore we begin by positing a candidate for a non-linear version of the field redefinition (11) (that mixes $g_{\mu \nu}$ and $\varphi_{\mu \nu}$ ) such that a consistent PM theory results from holding the redefined metric to a suitable fixed value:

$$
\left\{\begin{array}{l}
g_{\mu \nu} \rightarrow g_{\mu \nu}+\frac{6}{\Lambda} \varphi_{\mu \nu}+J_{\mu \nu}(g, \varphi) \\
\varphi_{\mu \nu} \rightarrow \varphi_{\mu \nu}+K_{\mu \nu}(g, \varphi) .
\end{array}\right.
$$

We take $J_{\mu \nu}$ and $K_{\mu \nu}$ to start at second order in $\varphi_{\mu \nu}$ so as to preserve the linear level choice (11). With this field redefinition, the CG action (10) reduces to that of a "matter" field $\varphi_{\mu \nu}$ coupled to a (dynamical) metric:

$$
S[g, \varphi]=\int \sqrt{-g}\left[-\frac{\Lambda}{6}(R-2 \Lambda)+\frac{6}{\Lambda} \mathscr{L}_{\mathrm{PM}}(\varphi, \nabla \varphi)\right]
$$

where $\mathscr{L}_{\mathrm{PM}}$ is the candidate PM Lagrangian. Its $\varphi_{\mu \nu}$ dependence is highly non-linear, with self-interactions coming from re-expressing all the original

\footnotetext{
${ }^{5}$ Indeed, the converse version of this procedure can be applied to produce cosmological gravity from CG for the full, non-linear theory: Examining the gauge transformations (13), we see that the PM background $\varphi_{\mu \nu}=0$ is preserved by diffeomorphisms but not Weyl transformations. Hence, setting $\varphi_{\mu \nu}=0$ yields a diffeomorphism invariant theory; performing this substitution in the action (10) yields cosmological Einstein gravity.
} 
metric dependence of the action (10) in terms of the shifted combination $g_{\mu \nu}+$ $\frac{6}{\Lambda} \varphi_{\mu \nu}+J_{\mu \nu}$. After making this expansion, we set $g_{\mu \nu}$ to any Einstein metric with cosmological constant $\Lambda$. This leaves us with the PM candidate

$$
S_{\mathrm{PM}}[\varphi]=\frac{6}{\Lambda} \int \sqrt{-g} \mathscr{L}_{\mathrm{PM}}(\varphi, \nabla \varphi)
$$

to be computed as an expansion in $\varphi_{\mu \nu}$ :

$$
\mathscr{L}_{\mathrm{PM}}=\frac{1}{4} \varphi^{\mu \nu}\left(\boldsymbol{G}_{\mu \nu}^{\rho \sigma}-\frac{2}{3} \Lambda \boldsymbol{F}_{\mu \nu}^{\rho \sigma}\right) \varphi_{\rho \sigma}+\sum_{n=3}^{\infty} \mathscr{L}_{\mathrm{PM}}^{(n)} .
$$

The absence of a term linear in $\varphi_{\mu \nu}$ follows from the linearized analysis and relies on the fact that $g_{\mu \nu}$ is now an Einstein metric. Furthermore, we notice that the arbitrariness of the field redefinition (14) encoded by $J(\varphi)$ and $K(\varphi)$ really amounts only to a field redefinition $\varphi_{\mu \nu} \rightarrow \varphi_{\mu \nu}+J_{\mu \nu}(\varphi)$ in the candidate action (16) when $J_{\mu \nu}=K_{\mu \nu}$. Moreover, examining the full non-linear form (10), we see that since $K_{\mu \nu}$ is at least second order in $\varphi_{\mu \nu}$, it only contributes to vertices at least quartic in the PM field. Hence, with the understanding that we only quote results for vertices up to possible $\varphi_{\mu \nu}$ field redefinitions, we may set $J_{\mu \nu}(\varphi)=0=K_{\mu \nu}(\varphi)$ without any loss of generality for our cubic order results.

Before presenting our explicit cubic vertices, let us show that there is no fully non-linear truncation of CG to an interacting PM theory. (This neither annuls consistency of the cubic vertices with respect to linearized gauge transformations, nor rules out any other ultimate theory of self-interacting PM fields.) To determine whether a truncation that takes $g_{\mu \nu}$ to be a fixed Einstein background is consistent, we must study the gauge invariances of the theory. The precise form of the underlying CG gauge transformations in terms of the redefined fields (14) is:

$$
\begin{aligned}
\delta g_{\mu \nu} & =\mathcal{L}_{\xi} g_{\mu \nu}-\frac{6}{\Lambda}\left[\nabla_{\mu} \partial_{\nu}+\frac{6}{\Lambda}\left[\left(g+\frac{6}{\Lambda} \varphi\right)^{-1}\right]^{\rho \sigma} \gamma_{\rho \mu \nu} \partial_{\sigma}\right] \alpha \\
\delta \varphi_{\mu \nu} & =\mathcal{L}_{\xi} \varphi_{\mu \nu}+\left[\nabla_{\mu} \partial_{\nu}+\frac{\Lambda}{3} g_{\mu \nu}+\frac{6}{\Lambda}\left[\left(g+\frac{6}{\Lambda} \varphi\right)^{-1}\right]^{\rho \sigma} \gamma_{\rho \mu \nu} \partial_{\sigma}+2 \varphi_{\mu \nu}\right] \alpha
\end{aligned}
$$

Here we have denoted the Christoffel symbols of $\varphi_{\mu \nu}$, covariantized with respect to $g_{\mu \nu}$, by

$$
\gamma_{\rho \mu \nu}:=\frac{1}{2}\left(\nabla_{\mu} \varphi_{\nu \rho}+\nabla_{\nu} \varphi_{\mu \rho}-\nabla_{\rho} \varphi_{\mu \nu}\right)
$$


Firstly observe that at leading order in $\varphi$, the choice of diffeomorphism parameter $\xi_{\mu}=3 \partial_{\mu} \alpha / \Lambda$ cancels the Lie derivative term $\mathcal{L}_{\xi} g_{\mu \nu}=\nabla_{\mu} \xi_{\nu}+\nabla_{\nu} \xi_{\mu}$ against the double gradient of the scalar parameter $\alpha$ in the metric variation. This is just a restatement of our linear result that the dynamical metric can be decoupled (at that order), leaving linear PM. Consistency of the non-linear truncation requires that there exist a choice of $\xi$ achieving this cancellation to all orders. This would determine the higher order terms in the variation of $\varphi$, leaving the PM action $S_{\mathrm{PM}}[\varphi]$ invariant. To establish a no-go result, we need only show that already no choice of $\xi$ achieves this cancellation for the next-to-leading (linear) order terms in $\varphi$ in the metric variation. Focussing on the linear part $\gamma_{\mu \nu}^{\rho} \partial_{\rho} \alpha$ of $\delta g_{\mu \nu}$ we immediately see that it can never be written as $\nabla_{(\mu} X_{\nu)}$, for any $X_{\nu}$ even on PM-shell. This establishes our claimed no-go result for truncating CG to a PM theory beyond linear order.

Finally, we turn to the computation of the cubic vertices. These, being guaranteed invariant under leading PM gauge transformations $\delta \varphi_{\mu \nu}=$ $\left(\nabla_{\mu} \partial_{\nu}+\frac{\Lambda}{3} g_{\mu \nu}\right) \alpha$, are candidate vertices for a putative non-linear self-interacting PM theory. The form of $n$-th order Lagrangian of the PM field determined by the field redefinition (14) (with $J_{\mu \nu}=0=K_{\mu \nu}$ ) can be obtained from the following correspondence,

$$
\begin{aligned}
\left(\frac{\Lambda}{6}\right)^{n+1} \sqrt{-g} \mathscr{L}_{\mathrm{PM}}^{(n+2)}= & \frac{n+1}{(n+2) !} \varphi_{\mu \nu} \delta_{\left.g\right|_{\varphi}}^{n+1}\left[\sqrt{-g} \mathcal{G}^{\mu \nu}\right] \\
& +\frac{\Lambda}{6} \frac{1}{n !} \delta_{\left.g\right|_{\varphi}}^{n}\left[\sqrt{-g} g^{\mu \nu} g^{\rho \sigma}\right]\left(\varphi_{\mu \rho} \varphi_{\nu \sigma}-\varphi_{\mu \nu} \varphi_{\rho \sigma}\right) .
\end{aligned}
$$

Here $\delta_{\left.g\right|_{\varphi}}^{n}$ signifies taking the $n$-th variation with respect to the metric and then replacing $\delta g_{\mu \nu}$ by $\varphi_{\mu \nu}$; the result is of $n$-th order in $\varphi_{\mu \nu}$. In the first line, we have used the fact that the first metric variation of the cosmological Einstein-Hilbert action produces the cosmological Einstein tensor $\mathcal{G}_{\mu \nu}$, which allows $(n+2)$ variations of that term to be combined with $(n+1)$ variations of the coupling of the cosmological Einstein tensor to the partially massless field in (10). If we evaluate the above interaction Lagrangians explicitly then, since they are given in terms of multiple variations of the Ricci tensor, the generic outcome for $\mathscr{L}_{\mathrm{PM}}$ is a two-derivative self-coupling of $\varphi_{\mu \nu}$, a curvature coupling and a potential for $\varphi_{\mu \nu}$.

We also note that multiplying the original CG action (2) by the dimensionfree combination $\Lambda^{-1} \kappa^{-2}$ of the cosmological constant and gravitational couplings and redefining the PM field $\varphi \rightarrow \Lambda \kappa \varphi$ gives, schematically, the canon- 
ically normalized action

$$
S \sim \frac{1}{\kappa^{2}} \int(R-2 \Lambda)+\int\left[(\nabla \varphi)^{2}+\Lambda \varphi^{2}\right]+\sum_{n=3}^{\infty} \kappa^{n-2}\left[\varphi^{n-2} \nabla \varphi \nabla \varphi+\Lambda \varphi^{n}\right]
$$

Now, let us focus on computing the cubic part $\mathscr{L}_{\mathrm{PM}}^{(3)}$ in (20). Note that since we work on an Einstein background, we may set $\mathcal{G}_{\mu \nu}=0$ (when it is not varied); also, since we only quote the vertex up to a possible field redefinition, at this order we may use the linear PM field equation, which can be written as $\delta_{\left.g\right|_{\varphi}} \mathcal{G}_{\mu \nu}+\frac{\Lambda}{3}\left(\varphi_{\mu \nu}-g_{\mu \nu} \varphi\right)=06$ Moreover, since the vertex is cubic in $\varphi_{\mu \nu}$, we may write

$$
\frac{6}{\Lambda} T^{\mu \nu}:=\frac{1}{3} \frac{1}{\sqrt{-g}} \frac{\delta S_{\mathrm{PM}}^{(3)}}{\delta \varphi_{\mu \nu}} \quad \text { and } \quad S_{\mathrm{PM}}^{(3)}=\frac{6}{\Lambda} \int \sqrt{-g} \varphi_{\mu \nu} T^{\mu \nu} .
$$

By construction, $S_{\mathrm{PM}}^{(3)}$ is invariant under the linear order PM gauge transformation (11) modulo the linear field equations. This guarantees that $T_{\mu \nu}$ obeys Noether identity

$$
\left(\nabla^{\mu} \nabla^{\nu}+\frac{\Lambda}{3} g^{\mu \nu}\right) T_{\mu \nu} \approx 0,
$$

in an Einstein background where $\approx$ denotes equality modulo the linear PM field equations.

It remains to explicitly compute $T_{\mu \nu}$. In fact, the cubic vertex itself is easily computed by hand by performing the variations of equation (20) for $n=1$. A computer aided computation [25] gives

$$
\begin{aligned}
T_{\mu \nu} \approx & \varphi^{\rho \sigma} \nabla_{\rho} \nabla_{\sigma} \varphi_{\mu \nu}+\frac{1}{2} \varphi_{\mu \nu} \Delta \varphi-\frac{4}{3} \varphi^{\rho \sigma} \nabla_{(\mu \mid} \nabla_{\rho} \varphi_{\sigma \mid \nu)}-\varphi_{(\mu}^{\rho} \nabla_{\nu)} \nabla_{\rho} \varphi \\
& +\frac{2}{3} \varphi^{\rho \sigma} \nabla_{\mu} \nabla_{\nu} \varphi_{\rho \sigma}+\frac{1}{6} \varphi \nabla_{\mu} \nabla_{\nu} \varphi+\frac{1}{6} g_{\mu \nu}\left(\varphi^{\rho \sigma} \nabla_{\rho} \nabla_{\sigma} \varphi-\varphi \Delta \varphi\right) \\
& +\nabla^{\rho} \varphi\left(\frac{3}{2} \nabla_{\rho} \varphi_{\mu \nu}-\frac{2}{3} \nabla_{(\mu} \varphi_{\nu) \rho}\right)-\frac{1}{3} \nabla^{\rho} \varphi_{\mu}^{\sigma} \nabla_{\rho} \varphi_{\sigma \nu}-\nabla^{\rho} \varphi_{(\mu \mid}^{\sigma} \nabla_{\sigma} \varphi_{\mid \nu) \rho} \\
& +\frac{2}{3} \nabla_{(\mu \mid} \varphi^{\rho \sigma} \nabla_{\rho \mid} \varphi_{\nu) \sigma}+\frac{1}{6} \nabla_{\mu} \varphi^{\rho \sigma} \nabla_{\nu} \varphi_{\rho \sigma}-\frac{1}{3} \nabla_{\mu} \varphi \nabla_{\nu} \varphi \\
& -g_{\mu \nu}\left(\frac{5}{12} \nabla^{\rho} \varphi^{\sigma \tau} \nabla_{\rho} \varphi_{\sigma \tau}-\frac{1}{2} \nabla^{\rho} \varphi^{\sigma \tau} \nabla_{\sigma} \varphi_{\rho \tau}+\frac{1}{12} \nabla^{\rho} \varphi \nabla_{\rho} \varphi\right) \\
& -\Lambda\left(\frac{1}{18} \varphi \varphi_{\mu \nu}+\frac{5}{9} \varphi_{\mu}^{\rho} \varphi_{\nu \rho}\right)+\Lambda g_{\mu \nu}\left(\frac{11}{36} \varphi^{\rho \sigma} \varphi_{\rho \sigma}-\frac{1}{36} \varphi^{2}\right) \\
& -\frac{2}{3} W^{\rho \tau}{ }_{(\mu}{ }^{\sigma} \varphi_{\nu) \tau} \varphi_{\rho \sigma}-\frac{2}{3} W^{\rho}{ }_{(\mu \nu)}{ }^{\sigma} \varphi_{\rho}^{\tau} \varphi_{\tau \sigma}-\frac{1}{3} g_{\mu \nu} W^{\rho \tau \kappa \sigma} \varphi_{\rho \sigma} \varphi_{\tau \kappa} .
\end{aligned}
$$

${ }^{6}$ Notice that the cubic vertex, therefore, schematically takes the form

$$
S_{\mathrm{PM}}^{(3)}=\delta_{\left.g\right|_{\varphi}} S_{\mathrm{PM}}^{(2)}+\int \varphi^{3},
$$

where $S_{\mathrm{PM}}^{(2)}$ is the leading order PM action and $\varphi^{3}$ denotes cubic potential terms in $\varphi_{\mu \nu}$. 
As a check, we verified that this $T_{\mu \nu}$ obeys the Noether identity (21) for the case of constant curvature $g_{\mu \nu}$.

We emphasize again that the consistency of the cubic vertices is independent of that of the higher order ones, implying that the vertex $S_{\mathrm{PM}}^{(3)}$ is valid beyond the context of CG. In fact, this vertex has been constructed in [12] where it has been shown that two-derivative self-interactions of PM fields exist only for $d=4$. We now see that CG underlies that result which is also consistent with the recent work of [13] where all consistent cubic interactions (not necessarily two-derivative ones) involving PM fields of generic spin were considered. There it was shown that for generic dimensions there are only two PM self-couplings involving at most four and six derivatives respectively. However, precisely in four dimensions, the Gauß-Bonnet identity reduces the maximal four-derivative coupling to a two-derivative one 7 .

\section{Conclusions}

We have revisited the well-trodden grounds of $d=4$ conformal, Weyl, gravity to explore the coupling of PM spin 2 fields to both fixed nontrivial curved backgrounds and dynamical gravity, as well as their self-interactions. The first result was that fixed Bach-flat backgrounds break the PM gauge invariance, and no natural background more general than Einstein was found in the space of all possible couplings to geometry and extensions of the gauge invariance for the linear PM theory. For dynamical gravity couplings, the attraction of CG was that it - at linearized level about dS - is the "difference" between a normal graviton and a PM one. However, upon insisting on a ghost-free model one must give up on any dynamical pretensions for the (ghost) graviton. This left the possibility that (just as truncating CG to its PM vacuum yields cosmological Einstein gravity) an appropriate truncation

${ }^{7}$ In fact, for constant curvature backgrounds, the Cotton-like tensor [10]

$$
F_{\mu \nu}{ }^{\rho}:=\nabla_{\mu} \varphi_{\nu}{ }^{\rho}-\nabla_{\nu} \varphi_{\mu}{ }^{\rho}
$$

is invariant under PM gauge transformations (11). (Strictly this version of the Cotton tensor is not the metric one, because the PM field is not the Schouten tensor, although in the underlying CG setting this is in fact the case.) Therefore any quartic derivative order, cubic vertex of type $\int(\nabla F) F F$ is PM invariant. In four dimensions, it should be possible to employ the Gauß-Bonnet identity to write this as a manifestly invariant cubic vertex quadratic in derivatives. 
of the dynamical graviton could allows CG to generate consistent PM selfinteractions. Here too we came up against the old problem, one that already occurs in trying to similarly extend higher-spin gauge theories: while it is possible to set up a - lowest order invariant - cubic self-interaction expressed as the coupling of the (quadratic) Noether current maintaining the initial Abelian invariance to the field amplitude, self-coupling inconsistencies set in at quartic order. That is, within the CG setting, one cannot extend the initial Abelian invariance to cover the next non-linear vertex. We illustrated this by exhibiting both the corresponding cubic terms and showing that there is no combination of fields, inert under (non-linear) PM transformations to the next order, truncating to consistent PM in a gravitational background. Although CG underlies cosmological Einstein gravity, it does not truncate to a non-linear "PM general relativity".

No-go theorems are notorious for their loopholes. Spin (2,3/2)-gravity and supergravity theories circumvent just such higher-spin pitfalls [26] while for (towers of) massive higher spins, string theory provides presumably consistent interactions and Vasiliev's theory describes interactions of towers of massless higher spins in (A)dS backgrounds [27]. Nonetheless, our results relying on $\mathrm{CG}$ as the underpinning of $\mathrm{PM}$ self-interactions seem quite robust. As stated in [12], "We have checked that it is impossible to proceed with quadratic approximation without introduction of higher derivative terms and/or some other fields." Moreover, also there, only a single PM cubic vertex was found; its agreement with the one generated by CG would suggest the absence of a self-interacting, two-derivative PM theory is a likely outcome.

One interesting feature of $\mathrm{CG}$ is that the PM field can be consistently turned off, leaving cosmological Einstein gravity (at least classically). In other words, without additional matter couplings, choosing initial conditions such that $\varphi_{\mu \nu}$ is zero at the initial time, then it will remain trivial, while the metric $g_{\mu \nu}$ can realize any Einstein solution [7]. We could also envisage a situation where the PM field $\varphi_{\mu \nu}$ is not strictly zero but rather nearly zero in some arbitrarily large time interval $t_{i} \ldots t_{f}$. Cosmology would then have approximate Einstein behavior for that epoch, while in the region $t \ll t_{i}$ or $t \gg t_{f}$, non-Einstein solutions could emerge. (The consequences for cosmological expansion with a partially conserved symmetric two index boundary operator were also considered in [8].) CG could then be used to generate transitions from a dS inflationary behavior of the cosmic scale factor to one controlled by the partially massless modes. Ghosts and loss of stability at 
early and late times may even be a useful/acceptable feature in this scenario.

A separate speculation is that gravity-like, or even self-interacting PMlike models for higher $s>2$, might be achievable by studying higher-spin versions of CG. Indeed, interacting conformally invariant higher-spin models that can be viewed as analogs of CG do exist [28, 29]. Perhaps a higher spin version of our approach could apply there.

\section{Acknowledgements}

We thank Hamid Afshar, Rod Gover, Daniel Grumiller and Karapet Mkrtchyan for fruitful discussions. EJ and AW acknowledge the ESI Vienna Workshop on Higher Spin Gravity. SD was supported in part by NSF PHY1064302 and DOE DE-FG02-164 92ER40701 grants. The work of EJ was supported in part by Scuola Normale Superiore, by INFN (I.S. TV12) and by the MIUR-PRIN contract 2009-KHZKRX.

\section{References}

[1] S. Deser and R. I. Nepomechie, Phys. Lett. B 132, 321 (1983); Annals Phys. 154, 396 (1984). Cited on pages 2, 10

[2] S. Deser and A. Waldron, Phys. Rev. Lett. 87, 031601 (2001) [arXiv:hepth/0102166]; Nucl. Phys. B 607, 577 (2001) [arXiv:hep-th/0103198]. Cited on page 2

[3] A. Higuchi, Nucl. Phys. B 282, 397 (1987); Nucl. Phys. B 325, 745 (1989); J. Math. Phys. 28, 1553 (1987) [Erratum-ibid. 43, 6385 (2002)]. Cited on page 2

[4] S. Deser and A. Waldron, Phys. Lett. B 508, 347 (2001) [arXiv:hepth/0103255]. Cited on page 2

[5] S. Deser and A. Waldron, Phys. Lett. B 513, 137 (2001) [arXiv:hepth/0105181]. Cited on page 2

[6] S. Deser and A. Waldron, Nucl. Phys. B 662, 379 (2003) [arXiv:hepth/0301068]. Cited on page 2

[7] J. Maldacena, arXiv:1105.5632 [hep-th]. Cited on pages 2, 3, 16 
[8] L. Dolan, C. R. Nappi and E. Witten, JHEP 0110, 016 (2001) [hepth/0109096]. Cited on pages 2, 16

[9] S. Deser and A. Waldron, Phys. Lett. B 603, 30 (2004), arXiv:hepth/0408155. Cited on pages 2, 10

[10] S. Deser and A. Waldron, Phys. Rev. D 74, 084036 (2006) [hepth/0609113]. Cited on pages 2, 15

[11] Y. M. Zinoviev, Nucl. Phys. B 821, 431 (2009) [arXiv:0901.3462 [hepth]]. Cited on page 2

[12] Y. .M. Zinoviev, Nucl. Phys. B 770 (2007) 83 [hep-th/0609170]. Cited on pages 2, 15, 16,

[13] E. Joung, L. Lopez and M. Taronna, arXiv:1203.6578 [hep-th]. Cited on pages 2, 15

[14] C. de Rham and S. Renaux-Petel, arXiv:1206.3482 [hep-th]. Cited on page 2

[15] K. S. Stelle, Phys. Rev. D 16, 953 (1977); S. Ferrara and B. Zumino, Nucl. Phys. B 134, 301 (1978); E. S. Fradkin and A. A. Tseytlin, Nucl. Phys. B 201, 469 (1982); Phys. Rept. 119, 233 (1985); R. J. Riegert, Phys. Lett. A 105, 110 (1984). Cited on page 3

[16] H. Lu, Y. Pang and C. N. Pope, Phys. Rev. D 84, 064001 (2011) [arXiv:1106.4657 [hep-th]]. Cited on page 3

[17] R. R. Metsaev, JHEP 1201, 064 (2012) [arXiv:0707.4437 [hep-th]]. Cited on page 3

[18] H. Lu and C. N. Pope, Phys. Rev. Lett. 106, 181302 (2011) [arXiv:1101.1971 [hep-th]]. Cited on page 3]

[19] S. -J. Hyun, W. -J. Jang, J. -H. Jeong and S. -H. Yi, JHEP 1201, 054 (2012) [arXiv:1111.1175 [hep-th]]. Cited on page 3

[20] M. Kaku, P. K. Townsend and P. van Nieuwenhuizen, Phys. Lett. B 69, 304 (1977). Cited on pages 3, 9 
[21] T.N. Bailey, M.G. Eastwood, and A.R. Gover, Rocky Mountain J. Math. 24 (1994), 1191. A.R. Gover, Adv. Math. 163 (2001), 206-257. Cited on pages 3 , 6

[22] A.R. Gover, J. Geometry and Physics, 60 (2010), 182, arXiv:0803.3510. Cited on page[

[23] S. Deser, R. Jackiw and S. Templeton, Ann Phys 140, (1982) 372; Phys. Rev. Lett. 48, (1982) 975. Cited on page4

[24] A. R. Gover, P. Somberg and V. Soucek, Commun. Math. Phys. 278, 307 (2008) [math/0606401 [math-dg]]. Cited on pages [6, (7)

[25] J. A. M. Vermaseren, "New features of FORM," [arXiv:math$\mathrm{ph} / 0010025]$. Cited on page 14

[26] H. Buchdahl, Nuovo Cim. 10, 96 (1958). Cited on page16]

[27] M. A. Vasiliev, Phys. Lett. B 243, 378 (1990). Cited on page[16

[28] A. Y. Segal, Nucl. Phys. B 664, 59 (2003) [hep-th/0207212]. Cited on page 17

[29] X. Bekaert, E. Joung and J. Mourad, JHEP 1102, 048 (2011) [arXiv:1012.2103 [hep-th]]. Cited on page[17 